\title{
VALIDACIÓN DE BRIQUETAS ELABORADAS CON CASCARILLA DE CAFÉ PARA EL REMPLAZO DE LEÑA EN LA PRODUCCIÓN DE ROSQUILLAS DE MAÍZ.
}

\section{VALIDATION BRIQUETTES MADE WHIT DESEEDED COFFEE FOR REPLACEMENT OF WOOD IN THE PRODUCTION OF CORN ROSQUILLAS.}

\author{
Luis María Dicovskiy\$ \\ Claudio Benito Pichardo ** \\ Aleyda Nohemí Rodríguez Zeledón t† \\ Bayardo Johexi Martínez Centeno $¥$ \\ Kiara del Rosario Rodríguez Espinoza $\$ \&$
}

RESUMEN: La investigación tuvo como propósito conocer la capacidad energética de las briquetas elaboradas con cascarilla de café para el reemplazo de leña en la producción de rosquillas. La eficiencia fue medida en distintas repeticiones evaluando la cantidad de agua a volumen constante que tanto la leña como las briquetas fueron capaces de evaporar. En un horno rosquillero tradicional se determinó que las briquetas tienen un $25 \%$ más de capacidad energética que la leña de ripio de pino y que esta no afecta la valoración sensorial en sabor y olor de las rosquillas de maíz. Concluyéndose que las briquetas de cascarilla de café son un producto apto para usar en hornos de rosquillas y que pueden ayudar a disminuir el uso de la leña.

Palabras Claves: briquetas; endocarpio; biocombustible; cascarilla de café; rosquilla

ABSTRACT: The research was aimed to know the energy capacity content of briquettes made of coffee husks to replace wood in the production of corn donuts. The efficiency was measured in different test series evaluating the amount of water at constant volume as both wood and briquettes were able to evaporate. In a traditional oven for baking corn donuts it was determined that the briquettes contain $25 \%$ more energy than gravel pine wood and that the use of briquettes made from coffee plants does not affect the taste and smell of corn donuts. As a conclusion it can be stated that coffee husk briquettes are suitable to use in ovens for baking corn donuts and can help reduce the amount of firewood.

Keywords: Briquettes, endocarp, biofuel, husk coffee, corn donuts

\footnotetext{
§ Sub-Director UNI-RUACS, Nicaragua, Luis.Dicovskiy@norte.uni.edu.ni - autor para la correspondencia.

** Coordinador de Carrera Ingeniería Agroindustria UNI-RUACS, Nicaragua, Claudio.Pichardo@norte.uni.edu.ni

†† Graduado Ingeniería Agroindustrial UNI RUACS, Nicaragua

¥ Graduado Ingeniería Agroindustrial UNI RUACS, Nicaragua

$\S \S$ Graduado Ingeniería Agroindustrial UNI RUACS, Nicaragua
} 


\section{INTRODUCCIÓN}

Nicaragua es un país productor de café principalmente en la zona norte (Jinotega, Matagalpa, Boaco, Nueva Segovia, Madriz y Estelí. Los residuos y sub-productos que genera este rubro constituyen una fuente de grave contaminación y problemas ambientales entre ellos está la cascarilla, que no es utilizada ya que es tratada como un desecho y la finalidad que le dan es incinerarla, en algunas ocasiones sin aprovechamiento de la energía producida por esta materia en combustión. Al mismo tiempo en la zona norte, son muy comunes los hornos para hacer rosquillas y pan dulce, los que utilizan leña que cada vez es más escaza y presiona más a los bosques locales. Esta Investigación se hizo con el objetivo de validar briquetas elaboradas con cascarilla de café para el reemplazo de leña en la producción de rosquillas.

\section{METODOLOGÍA}

Para evaluar la capacidad energética de las briquetas se montaron varios ensayos preliminares y finalmente un ensayo crítico con varias repeticiones. Cómo análisis estadístico se hizo un análisis de variancia y prueba de separación de promedios, para un diseño de bloques completos con repeticiones en el tiempo, de la variable "capacidad energética", medida por la cantidad de agua evaporada. Se usó cómo material testigo leña de ripio de pino.

Para conocer el efecto del uso briquetas en el sabor de rosquillas echas en horno, se hizo una prueba organoléptica comparando las briquetas contra leña de pino. Los resultados se analizaron estadísticamente cómo una prueba "t" basada en dos muestras independientes.

\section{RESULTADOS OBTENIDOS}

Con el objetivo de validar las briquetas elaboradas con cascarilla de café para el remplazo de leña en la producción de rosquillas se llevaron a cabo diferentes actividades y procedimientos, como determinación de las propiedades de la cascarilla de café, con el objetivo de identificar si la biomasa residual es apta para la elaboración de briquetas. Determinación de los porcentajes de humedad y ceniza, descripción del procedimiento artesanal para la elaboración de briquetas de cascarilla de café, posteriormente se realizaran varias pruebas de comparaciones entre leña, briquetas y cascarilla sin triturar con el fin de identificar cuál de estos productos presenta un mejor poder calorífico y combustión, se realizó una última prueba comparativa tomando como testigo siempre la leña esta vez las briquetas fueron quemadas para calentar el horno elaborando rosquillas y por último se realizó un análisis sensorial para determinar si las características en cuanto al sabor y olor de las rosquillas varían al ser horneadas con briquetas de cascarilla de café.

A continuación se presenta el cálculo de porcentaje de humedad, ceniza y descripción del proceso de elaboración y resultados obtenidos.

\section{Calculo del porcentaje de humedad de las briquetas}

Después de haber realizado la determinación de humedad a la cascarilla de café, se obtuvo un 7.9 \% dato que al compararlo con lo establecido según (Orozco, Cantarero, \& Rodríguez 
M) el cual refleja que debe ser de 7,6\% se puede indicar que es un poco más alto.

\section{Porcentaje de Cenizas.}

Se tomaron 6 muestras de $2 \mathrm{~g}$ de cascarilla de café en recipientes de porcelana cada una y posteriormente se introdujeron a la mufla a una temperatura de $500^{\circ} \mathrm{C}$ por un periodo de 2 horas y los residuos de material que quedaron después del quemado se pesaron en una balanza analítica Scout $600 \mathrm{~g}$ con una precisión de $0.01 \mathrm{~g}$, para determinar el porcentaje de ceniza generada. Según los datos calculados en dos pruebas se pudo determinar que la cascarilla presenta un $0.7 \%$ de ceniza material no combustible de dicha biomasa residual, el cual no presenta mucha diferencia de lo establecido por (Orozco, Cantarero, \& Rodríguez M) que es de $0.5 \%$.

\section{Proceso de elaboración de briquetas}

Primeramente se obtuvo la materia prima, posteriormente se trituró la cascarilla de café con el fin de obtener las partículas más finas y así lograr una mejor compactación. Luego se adicionó aglutinante, mezcla almidón más agua y se sometió a cocción hasta lograr un mezcla homogénea, posteriormente el aglutinante es mezclado con la cascarilla hasta lograr que este se adhiera a la cascarilla. La briqueta húmeda finalmente queda con la siguiente composición porcentual de peso: $65 \%$ cascarilla, 30\% agua y $5 \%$ almidón. Las briquetas pierden aproximadamente un $33 \%$ de humedad cuando son secadas. (Datos obtenidos con 7 repeticiones).

Como cuarto paso la mezcla es depositada en el molde y prensada ejerciendo presión a fuerza humana para lograr la compactación dejándola por un periodo de 10 minutos aproximadamente, luego la briqueta formada es extraída y se introduce en un secador eléctrico la cual presenta un peso de $450 \mathrm{~g}$ húmeda dejándola por 24 horas a una temperatura de $75{ }^{\circ} \mathrm{C}$, esto con el fin de eliminar el agua presente en el interior de la briqueta, luego del lapso del tiempo son ubicadas en sitios donde les da directamente el sol para completar el secado que este producto necesita quedando en un peso promedio de 320 g. En esta primera etapa se elaboraron 200 briquetas.

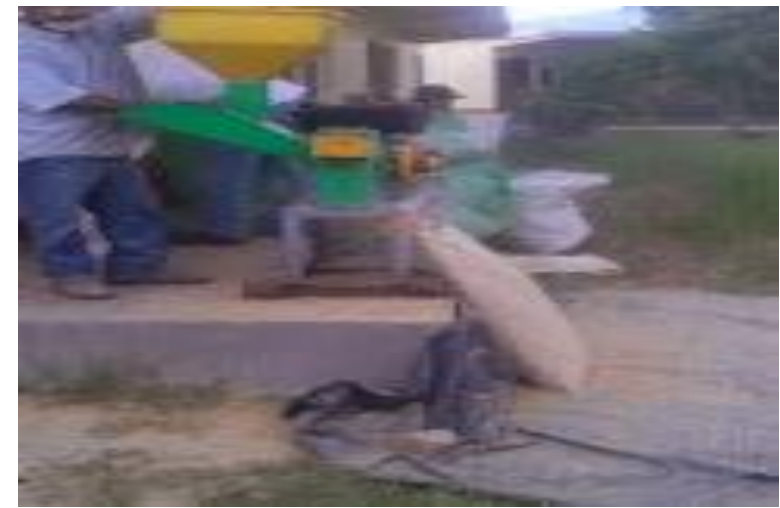

Fig. 1: Molienda de la cascarilla de café

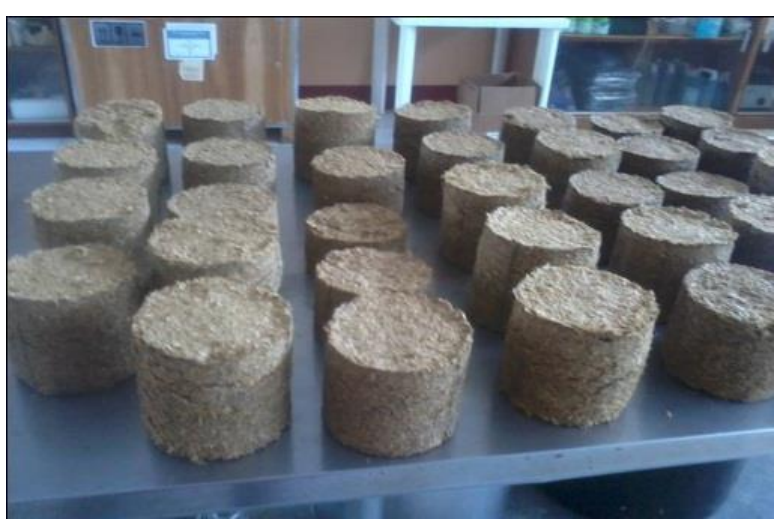

Fig. 2: Briquetas elaboradas 


\section{Pruebas preliminares realizadas a las briquetas para comparar su efectividad en una cocina industrial.}

Se realizó una prueba comparativa entre briquetas, leña de pino de ripio y cascarilla de café para determinar a pequeña escala la rentabilidad del producto que se está elaborando. Se usó una cocina económica, de lata, que funciona con cascarilla o aserrín. Primero se pesaron cantidades iguales de material: $2.2 \mathrm{~kg}$ leña $2.2 \mathrm{~kg}$ de briquetas y $2.2 \mathrm{~kg}$ de cascarilla de café. Luego se quemó la leña, cortada en trozos pequeños encendiéndolos en la cocina y se colocó un recipiente con 4 litros de agua para que este hirviera y medir cuanta cantidad se evaporaba y la cantidad de cenizas generadas, el mismo procedimiento se realizó con las briquetas y con la cascarilla de café sin compactar.

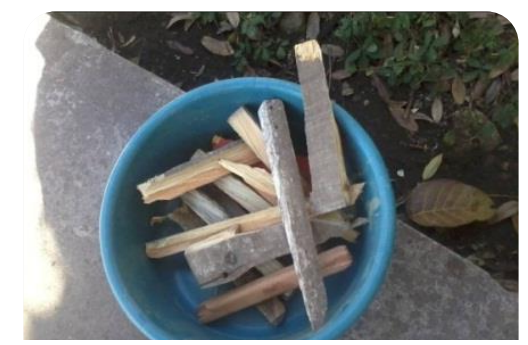

$2.2 \mathrm{Kg}$ de leña

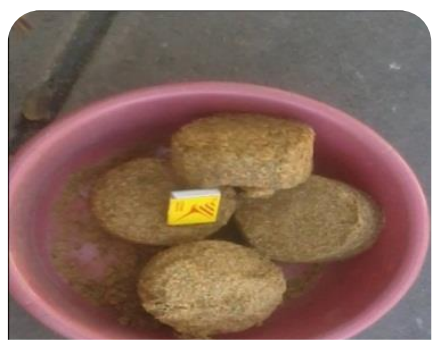

$2.2 \mathrm{~kg}$ de briquetas

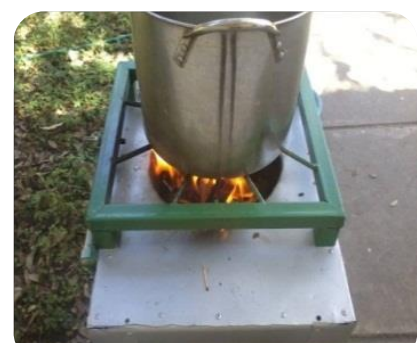

Quemado de briquetas

Fig. 3: Pesado y combustión de la leña y las briquetas

\section{Resultados obtenidos de las pruebas preliminares comparativas entre Briquetas, Leña y Cascarilla molida.}

Al realizar la prueba con leña de pino ripio, se obtuvo que esta duró encendida 1 horas con 10 minutos generando $247.8 \mathrm{~g}$ de ceniza y evaporando 0.6 litros de agua (15\%) de los 4 litros que se sometieron a calentamiento, llevándolos hasta una temperatura de $100{ }^{\circ} \mathrm{C}$. En la prueba con briquetas se determinó que estas duran encendidas 1 hora con 27 minutos, generando $160 \mathrm{~g}$ de ceniza y evaporando 1 litro (25\%) de agua de los 4 litros colocados. Un defecto observado fue que las briquetas se apagaron 4 veces, lo que obligó a utilizar carburante, diésel, varias veces. La prueba con cascarilla de café molida y sin compactar se pudo comprobar que el tiempo de encendido duro 1 hora con 10 minutos generando $300 \mathrm{~g}$ de ceniza y evaporando 1.4 litros de agua (35\%). A partir de estos datos se propuso hacer un experimento con briquetas que tuvieran un orificio en el centro, para quemar mejor.

\section{Experimento crítico realizado con las briquetas con orificios para comparar su efectividad energética en una cocina industrial.}

Se realizaron 5 pruebas comparativas con un diseño de bloques completos al azar, esta vez utilizando leña, ripio de pino, y briquetas a las cuales con un orificio en el centro con la finalidad que el encendido se produjera de adentro hacia afuera y el aire ventilara por ambos lados, utilizando $2.2 \mathrm{~kg}$ de cada producto en cada prueba de quemado y colocando 4 litros de agua para determinar la cantidad que se llega a evaporar. 


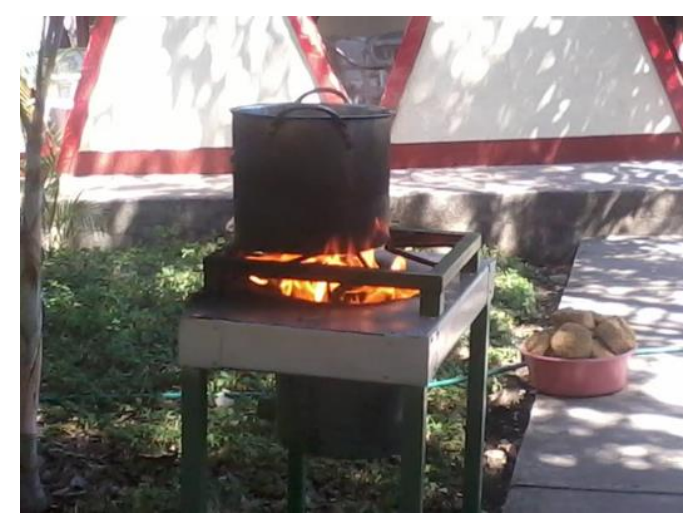

Fig. 4: Quemado de briquetas con orificios en el centro

Resultados obtenidos de un experimento crítico al comparar efectividad energética de las briquetas con orificios en el centro y leña.

Se pudo determinar que la briqueta con orificios funciona mejor que la leña de pino de ripio obtenido en un aserrío. Al comparar la capacidad de evaporación de agua, se identificó mejor resultado estadístico con las briquetas en su capacidad de evaporar agua.

A continuación se muestran los resultados estadísticos y un gráfico comparativo de los tratamientos, donde se realizó un análisis de bloques al azar, indicando que las briquetas poseen un mayor rendimiento energético.

\section{Análisis del experimento}

Tabla 1. Variación en la evaporación de agua

\begin{tabular}{|cccc|}
\hline Variable & $\mathbf{N}$ & $\mathbf{R}^{2}$ & Coeficiente de Variación \% \\
\hline Evaporación en $\mathrm{ml}$ de agua & 10 & 0.88 & 12.56 \\
\hline
\end{tabular}

El valor de $\mathrm{R}^{2}$ alto muestra que el experimento explica bien la variabilidad de los datos y el Coeficiente de Variación bajo que hubo poco error experimental

Tabla 2. Análisis de varianza de los dos tratamientos

\begin{tabular}{|lllllll|}
\hline F.V. & SC & gl & CM & F & p-valor \\
Modelo. & 112670 & 5 & 22534 & 5.88 & 0.0555 \\
\hline Tratamiento & 50410 & 1 & 50410 & 13.1 & 0.0222 \\
\hline Bloque & 62260 & 4 & 15565 & 4.06 & 0.1018 \\
\hline Error & 15340 & 4 & 3835 & & \\
\hline Total & 128010 & 9 & & & \\
\hline \multicolumn{5}{c}{ El valor "P" } & dicen que los tratamientos no son iguales.
\end{tabular}

Test: Duncan Alfa=0.05

Tabla 3. Test de Duncan del agua evaporada 


\begin{tabular}{|l|lcrr|}
\hline Tratamiento & ml evaporados & Sig. & \\
\hline Leña & 422 & A & \\
\hline Briqueta & 564 & & B \\
\hline
\end{tabular}

Medias con una letra común no son significativamente diferentes $(p<=0.05)$

La prueba de Duncan muestra que las Briquetas tienen más capacidad energética que la leña de pino evaluada, las Briquetas generan aproximadamente un $25 \%$ más de energía.

Gráfico 1. Resultados de evaporar agua con briquetas y leña.

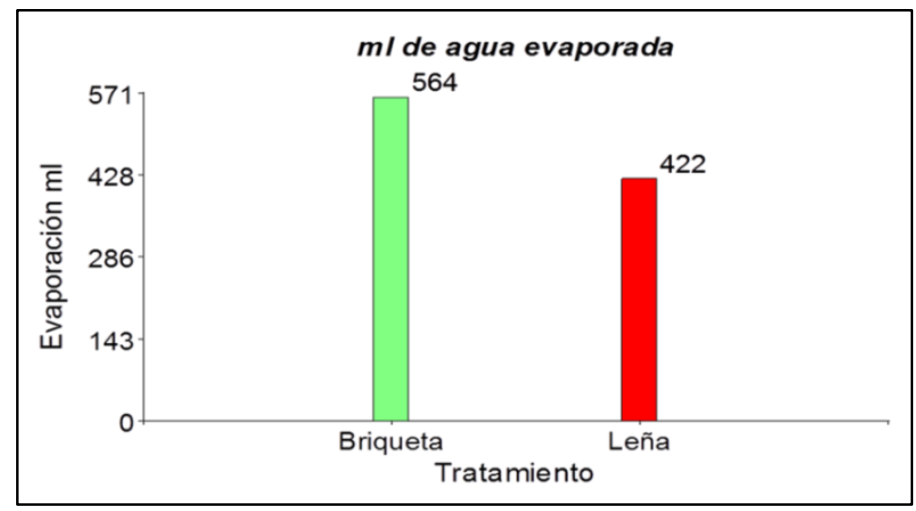

\section{Experimentación realizada en hornos rosquilleros}

Prueba para evaluar el rendimiento y eficiencia de las briquetas de cascarilla de café utilizadas en la elaboración de rosquillas. Estas pruebas se llevaron a cabo en un horno artesanal de barro en el municipio de San Juan de Limay departamento de Estelí, se realizaron dos pruebas a partir de una comparación un día se utilizó leña y al día siguiente se usó briquetas para calentar el horno pesando en iguales cantidades tanto la leña como las briquetas.

\section{Prueba con leña}

Se utilizaron 76 libras de leña para calentar el horno, el cual se encendió a las 8:00 am generando llamas abundantes, que a criterio de la dueña del horno creó una buena cantidad de brazas, transcurrido un lapso de tiempo de $1 \frac{1}{2}$ hora la llama disminuyo y se procedió a barrerlo con el objetivo de desechar las brasas y ceniza que produce la leña, luego se tomó la temperatura del horno el cual presento $270{ }^{\circ} \mathrm{C}$, exactamente a las 9:34 am, se introdujeron al horno las 15 cazuelejas con 10 unidades de rosquillas cada una dejándolas dentro del horno un tiempo máximo de 32 minutos hasta finalizar la hornada sacando las rosquillas, luego se tomó la temperatura del horno indicando que estaba a $170{ }^{\circ} \mathrm{C}$. 


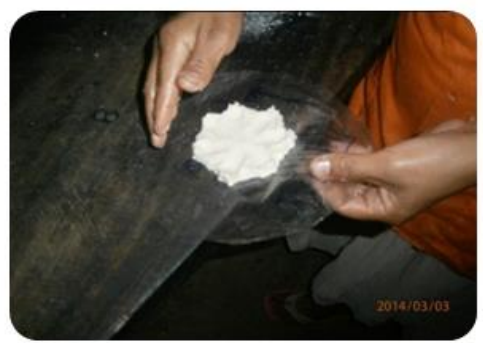

Elaboración de rosquillas

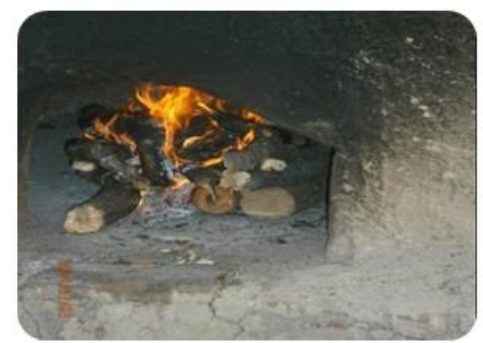

Llama que presento el quemado con leña en el horno

Fig. 5: Quemado de leña y elaboración de rosquillas

\section{Prueba con briquetas}

Se utilizaron 76 libras de briquetas para calentar el horno, iniciando a encenderlas a las 6:50 am introduciéndolas poco a poco el cual genero una llama grande que se mantuvo por un periodo de tiempo de 1 hora con 12 minutos disminuyo un poco pero la llama fuerte aun, se recurrió a barrer con las briquetas encendidas porque esta sobre calentado y las rosquillas se podían quemar, la escoba con que es barrido se quemó dos veces debido a que el horno estaba a altas temperaturas, luego se tomó la temperatura presentando $500{ }^{\circ} \mathrm{C}$, se esperó 20 minutos para que la temperatura bajara a $400^{\circ} \mathrm{C}$ y se introdujeron las 15 cazuelejas dejándolas dentro del horno 10 minutos hasta finalizar la hornada, con el fin de aprovechar el calor del horno se introdujeron 15 cazuelejas más a parte de las 15 provistas a hornear, posteriormente se tomó la temperatura y presento $145 \mathrm{C}^{0}$ luego de hornear 30 cazuelejas.
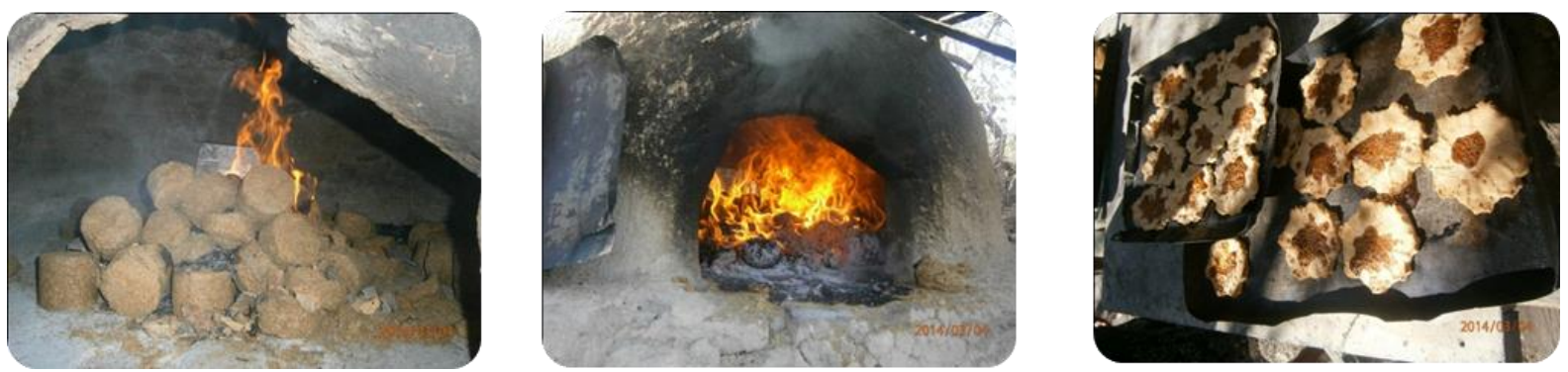

Fig. 6: Quemado de las briquetas y horneado de las rosquillas

\section{Aplicación de encuesta}

La encuesta se aplicó en la Universidad Nacional de Ingeniería, considerando tomando una población de consumidores de 20,000 personas, a través de la formula estadística de poblaciones finitas con un nivel de confianza de 95 y un margen de error del 10\% se calculó un tamaño de muestra de 96 personas a las cuales se les dio a degustar rosquillas hornadas con briquetas y rosquillas hornadas con leña como testigo, por medio de las cuales se pretendía que las personas pudieran determinar si existía diferencia de sabor y olor entre ambos productos. Se usó una escala con cinco valores, 1-5, siendo 5 la mejor valoración posible.

La población encuestada correspondió a personas de ambos sexos, El tipo de encuesta que se les aplicó fue estructurada, las preguntas ya estaban formuladas y el encuestado solo tenía que marcar la respuesta que se adecuaba a su preferencia. 


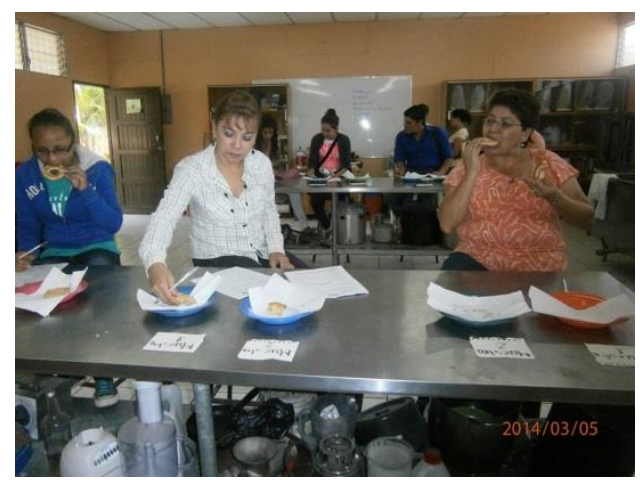

Fig. 7: Aplicación de encuestas

Se realizó una prueba "t" para muestras independientes para medir la evaluación sensorial, Sabor y Olor, de las rosquillas realizadas con fuego de Briquetas y de Leña. De los resultados obtenidos se pudo conocer identificar que no existe variación sensorial en cuanto a olor ni sabor entre ambas fuentes de calor, Briquetas y Leña.

Variable: Sabor - prueba: Bilateral “t” para muestras Independientes

Tabla 4: Evaluación del sabor de las rosquillas

\begin{tabular}{|lrlr|}
\hline Generalidades & Grupo 1 & Grupo 2 & \\
\hline n & Briqueta & Leña & 96 \\
\hline Media & 96 & 3.88 \\
\hline p Hom Var & 3.82 & \\
\hline "t" & 0.0114 & \\
\hline p-valor & -0.41 & \\
\hline
\end{tabular}

Prueba T para muestras Independientes

Tabla 5: Evaluación organoléptica del olor de las rosquillas Variable: Olor - - prueba: Bilateral

\begin{tabular}{|c|c|c|}
\hline Generalidades & Grupo 1 & Grupo 2 \\
\hline & Briqueta & Leña \\
\hline n personas & 96 & 96 \\
\hline Media & 3.86 & 3.88 \\
\hline pHomVar & 0.3038 & \\
\hline $\mathbf{T}$ & -0.08 & \\
\hline p-valor & 0.933 & $\mathrm{H}_{0}$ Promedios iguales \\
\hline
\end{tabular}

Gráfico 2. Resultados de la evaluación sensorial de elaborar rosquillas con briquetas y leña. 


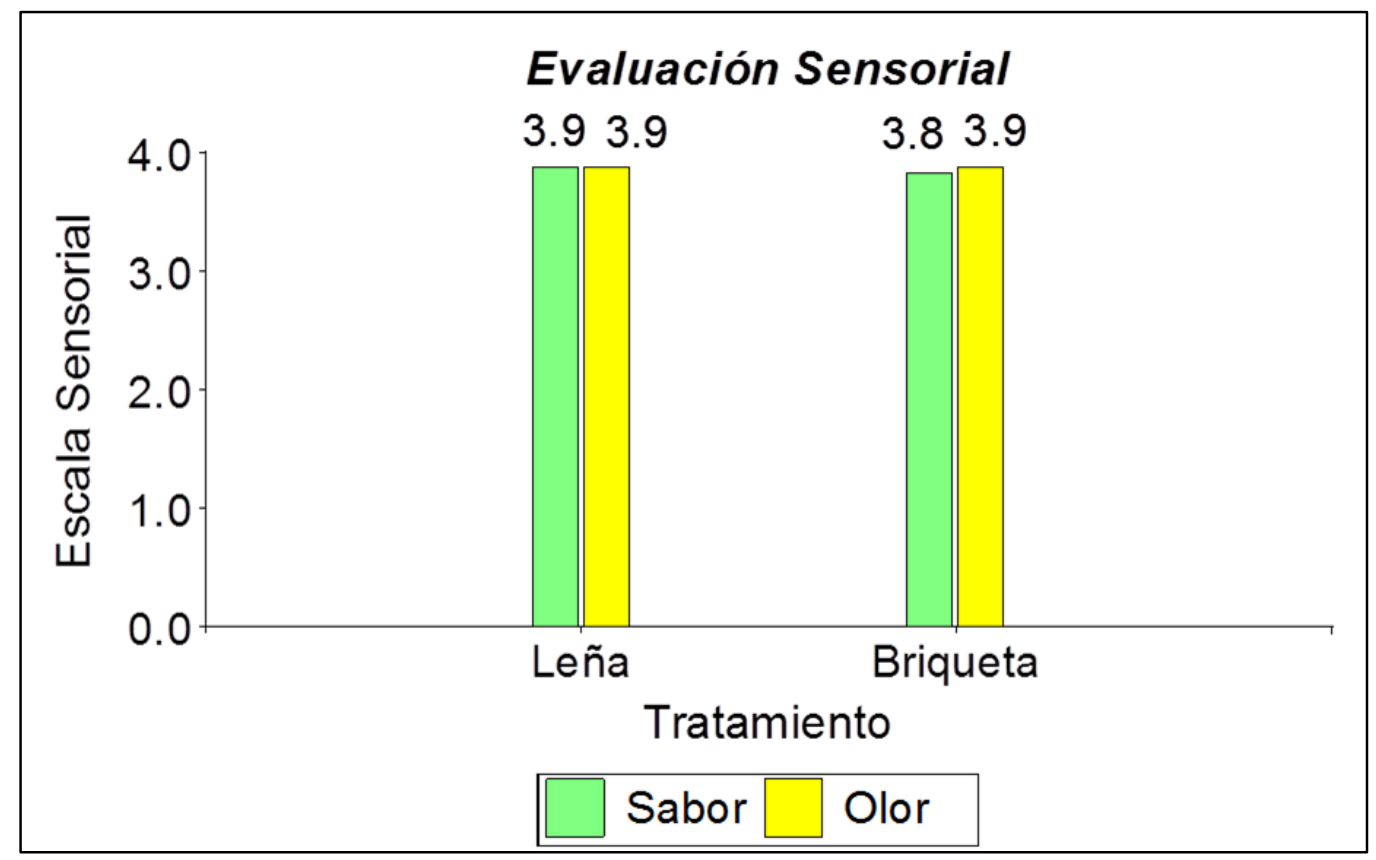

\section{CONCLUSIONES}

Del primer experimento preliminar realizado se observó que las briquetas duran un poco más tiempo encendidas que la leña y la cascarilla suelta sin compactar, siendo su llama un poco más lenta, y que la leña de ripio se quema más rápido, se eligió este tipo de madera por ser la que es más utilizan las rosquilleras. Las briquetas evaporaron más agua, que la leña y menos que la cascarilla sola. En cuanto a la generación de cenizas se determina que las briquetas generan poca ceniza y bastante calor comparado a la leña y cascarilla de café. Con la salvedad que este experimento se hizo en una cocina ecológica, diseñada para quemar cascarilla, con ventilación por la parte lateral inferior y superior central del recipiente, efecto que no ocurriría en un horno de ladrillo, donde la cascarilla no podría quemarse por falta de ventilación en la base del montículo que se forma, le faltaría el efecto de chimenea que hizo la cocina.

Al realizar un experimento crítico de comparación entre briquetas de cascarilla de café con orificio central en cada una de ellas y con leña, se determinó que las briquetas tienen un 25 \% más de capacidad energética para evaporar agua, más duración de encendido y menos porcentaje de ceniza en comparación con la leña, lo mismo se identificó en la experimentación realizada en el horno al producir rosquillas, ya que al quemar las misma cantidad de leña y briquetas, este último producto hizo sobre pasar la temperatura de $400^{\circ} \mathrm{C}$ en cambio la leña alcanzo como máximo $270^{\circ} \mathrm{C}$. Las briquetas, al calentar tanto el horno, se pudo duplicar la capacidad para hornear rosquillas.

Por otra parte se tiene que el producto es apto como reemplazo de leña en la producción de rosquillas, ya que no afecta la valoración sensorial de este producto alimenticio.

Se concluye que las briquetas son producto apto para usar en hornos de rosquillas, que pueden ayudar a disminuir el uso de leña, lo que significará un menor despale de bosques, más captura de carbono y además se le dará un valor agregado a un desecho orgánico que los beneficios secos no utilizan eficientemente. 


\section{REFERENCIAS}

Orozco, S. C., Cantarero, P. V., \& Rodríguez M, J. F. (s.f.). Tratamiento de residuos del cafe PROMEcafe_ILCA PELCCE ICAFE. Matagalpa,Nicaragua: PROMECAFE, ILCA, PEICCE e ICAFE.

Luis María Dicovskiy Rioboo: Es Graduado de Ingeniero
Agrónomo de la Universidad Nacional de Rosario UNR, Argentina.
Tiene especialización en mejora vegetal en el Instituto de Altos
Estudios del Mediterráneo, Zaragoza, España., master en
estadística e investigación de operaciones otorgadas por la
Universidad de Valencia, España, y master en investigación social
cualitativa, otorgado por la Universidad Politécnica, UPOLI,
Nicaragua. Es profesor de las asignaturas de estadística,
seminario metodológicos, Ingeniería pos cosecha y control
estadístico de la calidad en la sede Regional del Norte de la
Universidad Nacional de Ingeniería de Nicaragua.

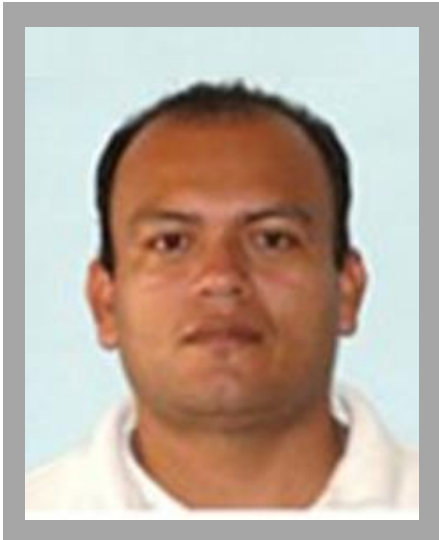

Claudio Benito Pichardo: Es Graduado de Ingeniero en Alimentos en la Universidad Nacional Autónoma de Nicaragua UNAN-León, Nicaragua. Tiene maestría en procesamiento de Alimentos, otorgado por la Universidad Nacional de Ingeniería. Docente de las Asignaturas procesos Agroindustriales III, procesos Agroindustriales IV, Bioquímica, Buenas prácticas Agrícolas y de Manufactura en la sede Regional del Norte de la Universidad Nacional de Ingeniería de Nicaragua. 